\title{
A PHOTOGRAPHIC STUDY OF THE ELECTRIC ARC.
}

BY N. H. BRown.

T F a photographic plate or film is moved rapidly in front of a lens 1 or camera, in such a position that the image of some object is thrown on the sensitive part of the plate, an impression will be produced on the plate which is a more or less perfect history of the changes that took place in that object while the plate was moving. If the image is quite broad there will necessarily be much blurring of the plate, causing lack of definition.

Various attempts have been made to learn something about arc lights by photographs taken in this way when the arc was undergoing some variation. ${ }^{1}$ The pictures obtained have been interesting, but have lacked the definition which usually characterizes a photograph. Naturally, the alternating current has been best adapted to this study, for the changes are fairly uniform and frequent. The advent of the alternating current enclosed arc lamp has furnished an arc that is much steadier in its action, and, therefore, more uniform in its variations than any previous arc, and thus better adapted to this work. For this reason most of the work described in this paper was carried out with the aid of such a lamp.

We shall consider the various types of photographs connected with this work, discussing each as it is described, instead of describing all and following with a general discussion.

Pictures numbered one (See Plate I) were taken on a sliding plate without a screen, as shown in diagram I of Fig. I. We find here a marked symmetry, not only between the various pictures but between the parts of one picture. Each light spot is like the second, fourth, etc., from it. On closer inspection we notice that if any spot were turned over it would be like its next neighbor. The front end of each light portion is not square across the picture but makes a

1 See La Lumiere Electrique, I89r, pp. 555. Also Proceedings of the American Institute of Electrical Engineers, I891, Vol. 8, pp. 214. 
very appreciable angle. This seems to indicate not only that the arc was out part of the time, but also that the lighting up did not take place across the whole arc space at the same instant, having started from one side one time and the opposite side the next time. A simple calculation, depending upon the velocity of the plate and the slope of the light front, shows that this light travelled across the arc with a velocity of about 300 inches per second from the negative carbon toward the positive.

In one or two of these pictures we can see slight indications that there is another light front advancing from the positive toward the

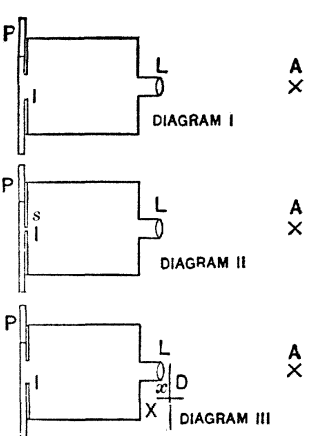

Fig. 1. negative, but we shall see this much more clearly from another set of pictures. The back end of the light space seems to be away from both carbons, as though the light died out near the carbons first, and later near the center of the arc space. This seemed strange and so led to the fear that the shapes of the front and back ends of the bright portion of the plate were due, in some way, to the shape of the arc itself. For instance, suppose the arc had been conical as it was first lighted, and that the lighting was practically instantaneous. Suppose also that as the arc commenced to decrease in intensity it changed into a globular form. We could then account for the peculiar shape of the arc pictures without supposing that appreciable time was required to light the arc. In order to test this point pictures were taken with a narrow slit placed just in front of the image of the arc on the plate. If a screen, which has a very narrow slit in it, be placed very close to the plate as it is moved in front of the lens, as shown in Fig. I, diagram II, so that most of the image is cut off, we may get a fairly clear image of the small part that is left. The results obtained in this way are shown by pictures numbered 2, Plate II. From these it is evident that there were two light fronts, one from each carbon; and that one of these light fronts started about $\mathrm{I} / \mathrm{IOOO}$ of a second before the other. Their slopes show that they travelled with different velocities, the one from the negative side moving about three hundred inches per second, while the other moved about five hundred inches per second. 
Some of these plates were under-developed to bring out differences of density more clearly. The slit being left less than $\frac{1}{64}$ of an inch wide the outer contour of the arc could have had little to do with the shape of the pictures. Each consecutive vertical section of the picture must be the record for successive intervals of time, of that particular vertical section of the arc which was thrown on the slit.

Blondel and McMynn, in the articles already referred to, each showed pictures showing some of the characteristics just mentioned, but the effect was so slight that neither of them noticed and discussed them. By comparing their pictures with these we find from similarity that we are able to easily tell which of the terminals was positive at any given instant, which way the plates were moving, etc.

These conclusions were so striking that it seemed well to get some other results that would verify them, and to attempt to explain why we should get such results. We have assumed that the pictures on Plate II. were of some section of the arc. If we could only take pictures of the whole arc at frequent intervals we could hope for confirmation. But to secure such pictures of the arc as it is at any instant it is necessary, as with everything else, that the movement of the plate be very slight during any exposure, and that the movement or change in appearance of the arc should be very slight. In order to separate the various parts of an alternation as much as possible on the plate it seemed necessary to run the plate at a speed of 40 to 50 feet per second. As a movement of the plate of one-hundredth of an inch or more during an exposure would injure the definition of the picture the time of exposure must be very small. The pictures numbered three (Plates I) were taken with the apparatus arranged to give rapid exposures of the whole arc, as shown in Fig. I, diagram III. When the disc D was revolved about its axis I 5,000 times per minute each of the four slits would cause an exposure which lasted about $\frac{1}{25} \frac{1}{00} \overline{0}$ part of a second. There would be I,O0O exposures a second, or about eight images for each complete period (the frequency was about I25).

The pictures numbered three were taken with various velocities of the disc from 7,000 revolutions per minute up. Here we find the arc in all possible conditions, and the conclusions reached by 
our former method are fully verified. The picture $3 \mathrm{D}$ was taken when a magnet was held close to the arc. Here the arc is deflected to one side or the other depending on the direction in which the current is flowing. At times the last end of the light from one-half period has not faded away when the new light front for the next period has arrived. With the magnet near, the fading light of one period is driven in one direction while the new light formation due to the next period is sent in the opposite direction. They are thus separated in such a way as to show clearly to which period they respectively belong.

I examined the arc for plane polarization by setting a Nicol prism in front of the arc and taking a number of pictures, rotating the prism a few degrees for each till $90^{\circ}$ of arc had been covered. No effect whatever was noted.

Nothing thus far has shown that the formation of the two light fronts on the opposite ends of the arc in the way we have described is not a function of the form of the current curve. To determine this point pictures were taken of a very slow period arc $(22$ per second). One of these is shown in number 4. They seem to be like the other pictures in all characteristics except that everything is exaggerated somewhat. This current was of the "true sine wave" form, while the others were "peaked." Thus the characteristics are at least not very sensitive to small changes in the wave form. To further vary the type of current a direct current was sent through the arc. This was short circuited during part of the time while the plate was moving across in front of the arc. Before the plate had passed entirely across the short circuit was broken, relighting the arc. This acted in such a way on the lamp mechanism as to rebreak the arc. Thus we had on our plate: First a steady arc, second the arc broken almost absolutely instantaneously, third the arc formed again with very great suddenness and with slightly higher current value than formerly, fourth the arc broken by the separation of the carbons. These pictures are shown numbered 5. When the arc is first broken a quite abrupt line is visible, showing a very sudden diminution of the light. The fading light from that line forward is as it was with the alternating arc. When the arc is reformed its difference from the alternating arc is very marked. At 
[VoL. VII.

first sight it seems to be almost square across the end, but, on closer examination, it shows that the light did not reach the center quite as soon as it left the sides. The difference in time is very slight, however, as compared with the difference in the case of the alternating current. Then, too, with the alternating current the negative carbon gives out light first while here the positive is slightly ahead. The picture number 6 was taken in the same way as its predecessors, except that a very fortunate accident occurred. The second extinction of the arc took place so quickly after the arc formed the second time that the light front only started to form by the time the arc was extinguished. As can easily be seen from the picture the carbon was already moving away when the arc was formed. This leaves no doubt whatever that the light front starts near the positive carbon.

With these data it seems best to advance one or more hypotheses which will account in a more or less satisfactory manner for the phenomena. First, let us suppose that this source of light is due to ions moving across the arc very much as they move in electrolysis; either that the particles actually move with a velocity of the light front, or that the space being filled with particles a wave of change passes with that velocity. We would then suppose that the particles could move from the negative carbon more readily than from the positive. This would be what we would expect from the fact that most of the electromotive force (necessary to send a current through the arc) is overcome very close to the positive carbon. Under these conditions a particle thrown off from the positive carbon would move with a greater velocity than one thrown from the negative. This supposition would account also for the difference in slope on the last end of the light space as is shown in several pictures, and for the finishing of the light at the center. It would also account for the fact that the light front from the positive carbon is stronger than that from the negative.

The first serious obstacle confronting this hypothesis is the pictures of the interrupted arc shown in numbers 5 and 6 . In these the start comes first from the positive side, and the finish is at practically the same point. Here the successive arcs are in the same direction. If now we can advance any hypothesis which will 
base the point of starting of the light front on the polarity during the preceding illumination the difference in direction between the alternating and direct arcs will be accounted for. Let us suppose that particles being in the space between the carbons the light is produced by heating them. If a current which is very weak at first starts across the arc space it is reasonable to suppose that while it is a weak current its main heating effect will be at the carbon surfaces. Now, if one of the carbons (the negative) is much hotter than the other, that one should show the first effect; hence the light would start from the negative. Then as the current continued and increased the positive carbon would reach and quickly pass the temperature of the negative, and thus there would be a stronger effect from the positive than from the negative, and thus too, it would move faster. And as the current became greater the direct effect of the current on the gas would become greater. When the arc stopped we should suppose that the solid carbon would dispose of its heat much more rapidly than the gas. This would cause the light to last longer toward the center. This would account, too, for the tardiness with which the light fades out near the positive side. With the interrupted arc, according to this hypothesis the sudden falling off in the light at the instant of cutting out the arc would be due to the fact that the current was not flowing, and, therefore, there was no source of heat in the gas. The further gradual cooling would be due to radiation or other causes, as in the alternating arc. Here, too, it is natural that the gas should cool nearer to the carbons. When the arc is re-starting it is started with full or more than full current, and the effect of the arc directly on the gas is much greater than when the current is small; hence, we naturally expect the hottest part to glow first and our light starts near the positive carbon, but not quite at the carbon, as shown in number 6 .

Photograph No. 7 was taken with the image of a vertical arc thrown on a plate moving vertically. Here we find, in general, that the light moves out horizontally under the influence of a magnet near the arc, and that the direction of this movement depends on the direction of the flow of the current in the arc, but we failed to get sharp definition. Plate number 8 was taken in the same way, except that a very narrow slit was placed horizontally as close 
as possible to the plate and the speed of the plate was increased. This gives good definition and shows also that while the whole source of light is moved to one side it is not moved uniformly, and thus the bright band is broadened out. This broadening out, moreover, does not leave the light uniform throughout the whole band, but separates it into several different bands, as though the current flowing across the arc consisted of waves of distinctly different frequencies or wave-lengths, or was composed of several series of currents where each current of a series differs from the next to it by a very small amount.

This band would then correspond to a spectrum in which some broad lines were wanting.

It would be reasonable to assume in this connection that this electric spectrum was in some way a function of the winding of the dynamo or the shape of the field magnets and that it may be modified somewhat by the transformers or inductive resistance through which the current passes.

No definite statement can be made regarding this, however, for all of the pictures so far have been taken under too nearly the same conditions to bring out such differences. At first thought it seems strange that the consecutive alternations do not appear symmetrical, but this is easily explained when we know that the slit is very close to the image of one of the carbons and a relatively long distance from the other.

Realizing the incompleteness of the work described I have yet ventured to report it, hoping for opportunity in the near future for fuller investigation.

After the copy for this article was in the printer's hands my attention was called to a paper in the June, I 898, Proceedings of the American Academy of Arts and Sciences (Vol. XXXIII., No. I 8) by Henry Crew and Olin H. Basquin on "The Sources of Luminosity in the Electric Arc." They have used the same general method as that employed in preparing this paper except that their pictures are composites made through a comparatively wide slit while those here shown are for single exposures through a narrow slit. Their conclusion that the persistent luminosity is chemical or thermal and not electrical would correspond with the work here presented.

Physicai. Laboratory of Cornell University. 
PHYSICAL REVIEW, XXXVIII.

(To face page 216.)

PLATE $\mathrm{I}$.
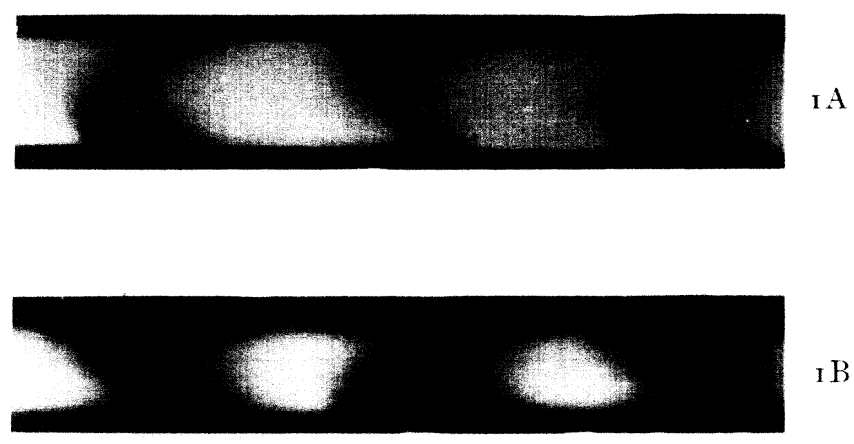

IB
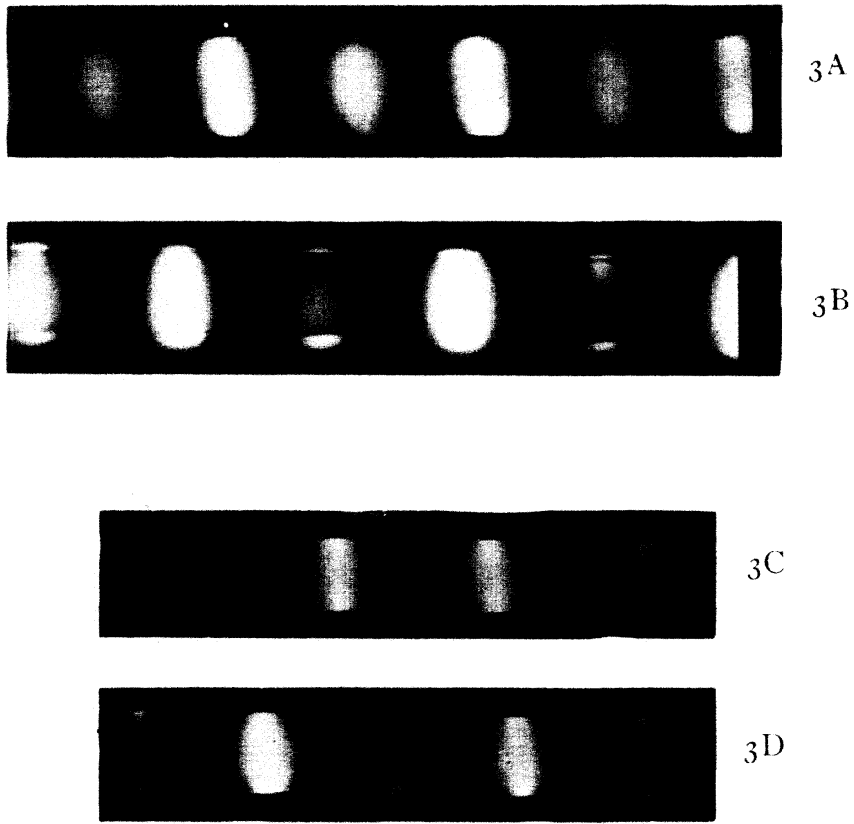

$3^{\mathrm{D}}$

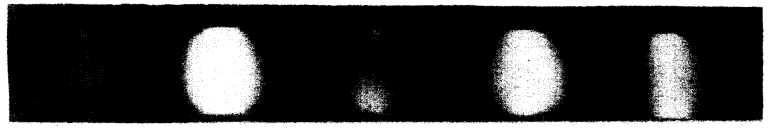

${ }_{3} \mathrm{E}$

BROWN. A PHOTOGRAPHIC STUDY OF THE ELECTRIC ARC. 
PHYSICAL REVIEW, XXXVIII.

PLATE II.

(To face page 216 .)
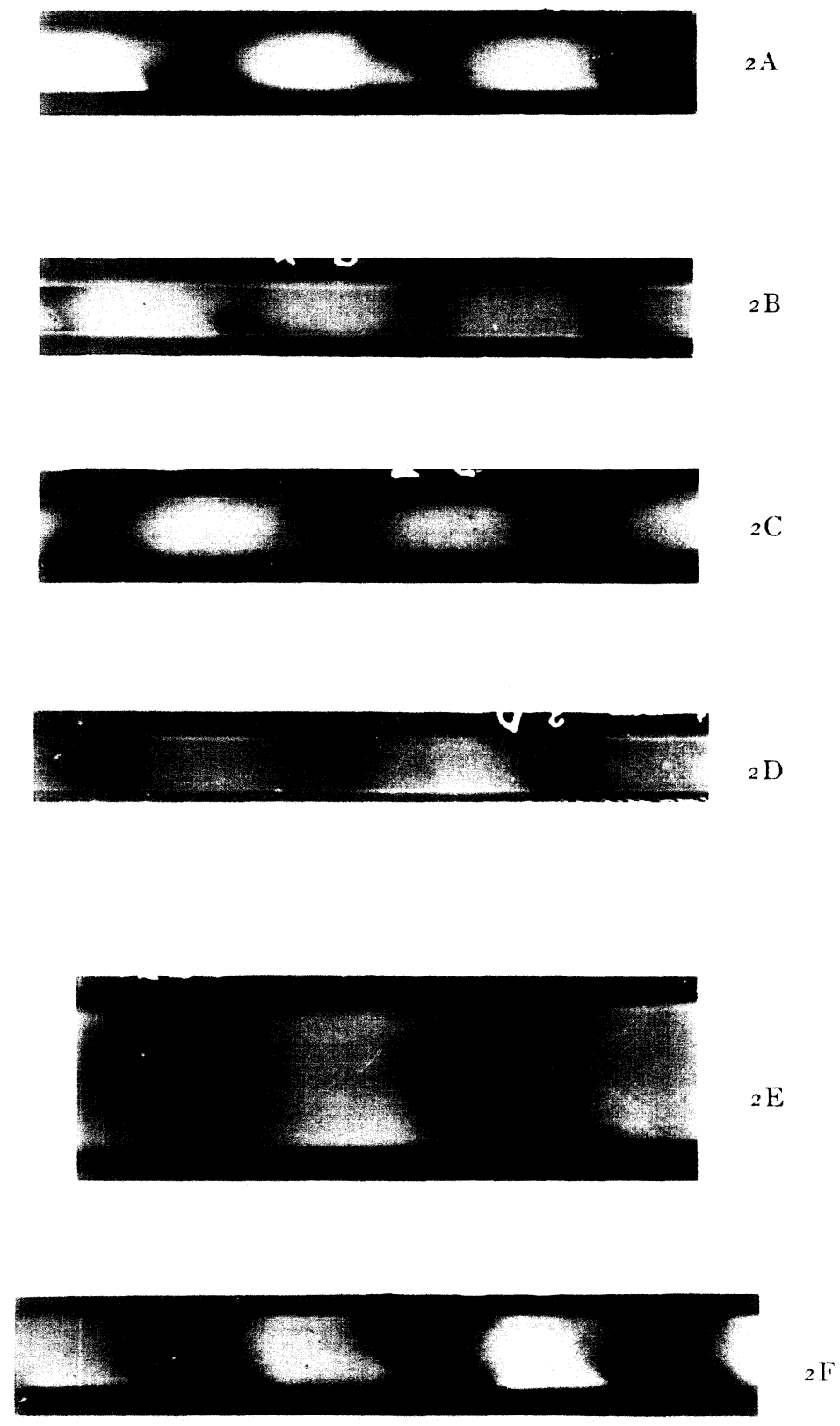

BROWN. A PHOTOGRAPHIC STUDY OF THE ELECTRIC ARC. 
PHYSICAL REVIEW, XXXVIII.

(To face page 216.)

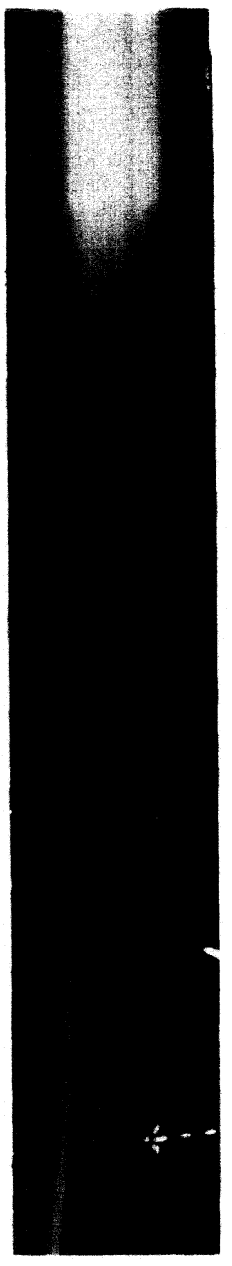

6

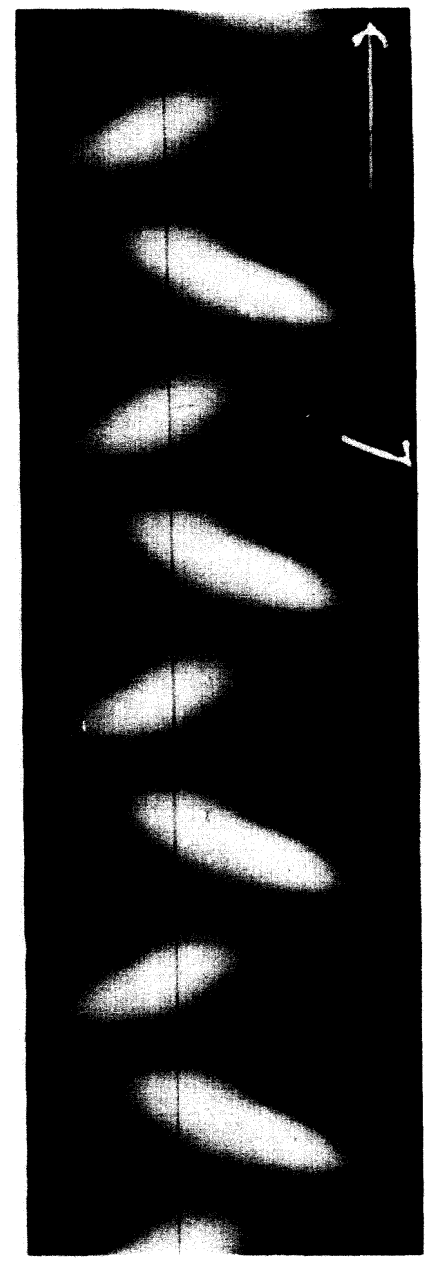

7

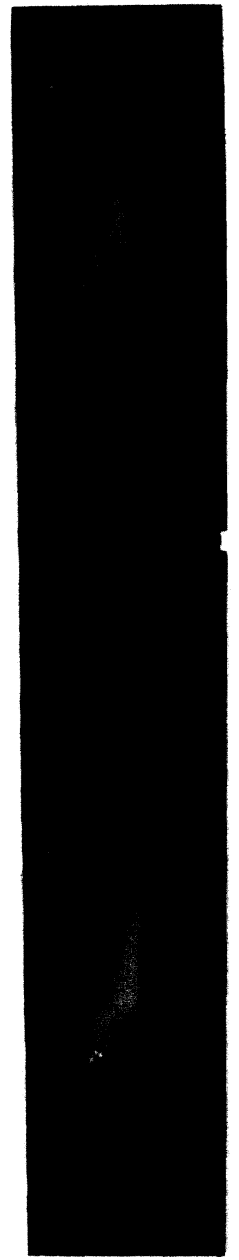

8
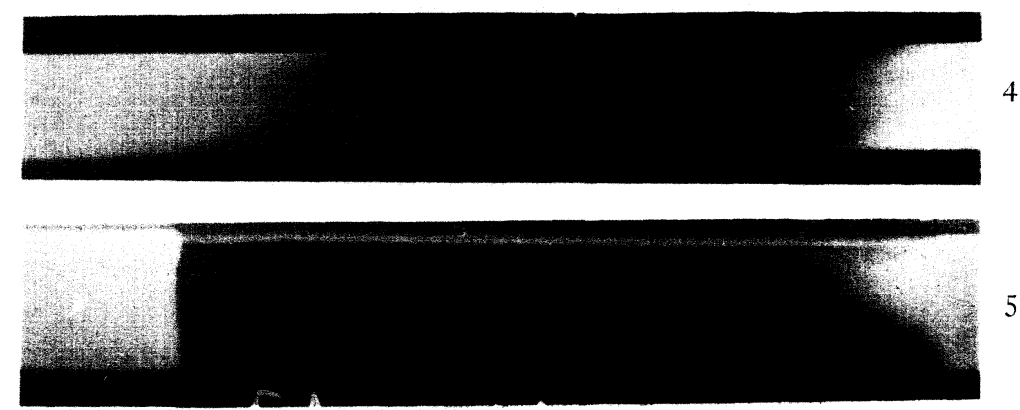

BROWN. A PHOTOGRAPHIC STUDY OF THE ELECTRIC ARC. 
PHYSICAL REVIEW, XXXVIII.

PLATE I.

(To face page 216.)
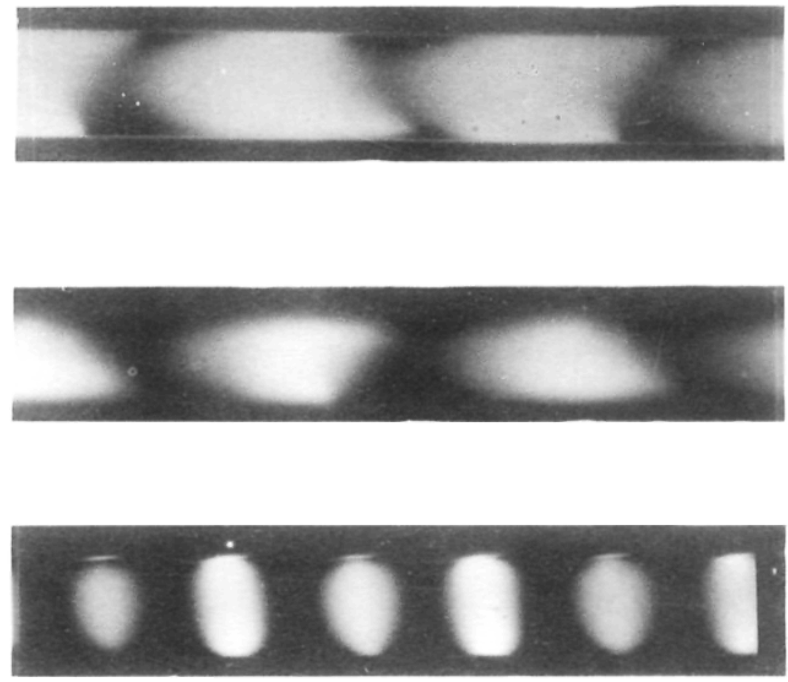

$3^{\mathrm{A}}$
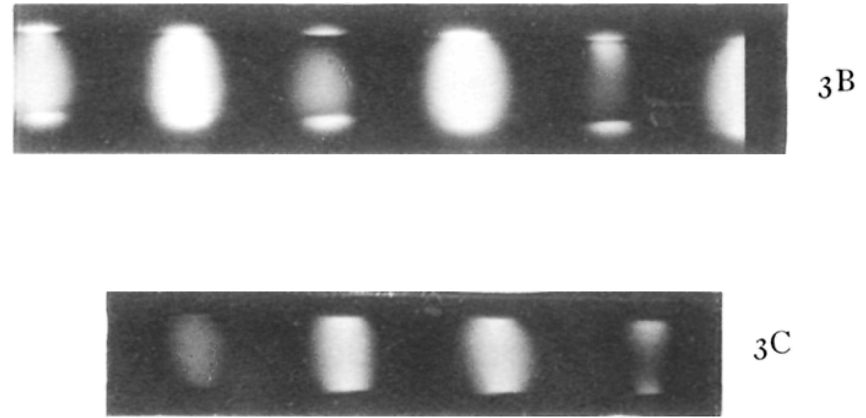

${ }_{3} \mathrm{C}$

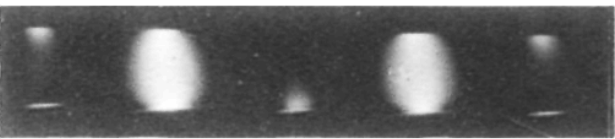

${ }_{3} \mathrm{D}$

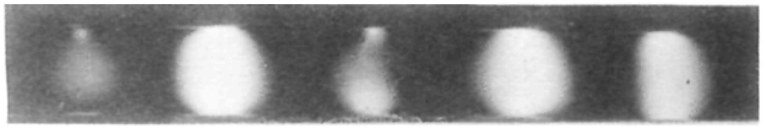

${ }_{3} \mathrm{E}$

BROWN. A PHOTOGRAPHIC STUDY OF THE ELECTRIC ARC. 
PHYSICAL REVIEW, XXXVIII.

(To face page 216.)
PLATE II.

$2 \mathrm{~A}$

$2 \mathrm{~B}$

${ }_{2} \mathrm{C}$

$2 \mathrm{D}$

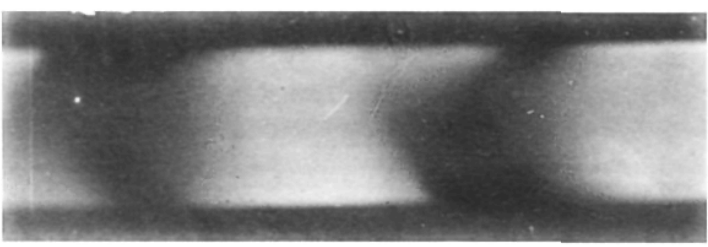

$2 \mathrm{E}$

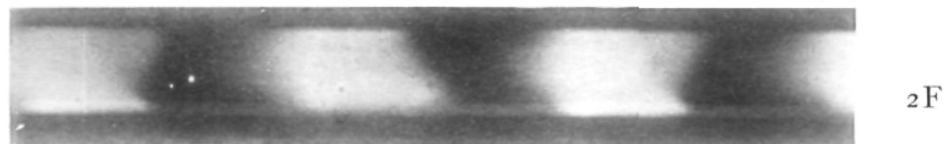

BROWN. A PHOTOGRAPHIC STUDY OF THE ELECTRIC ARC. 
PHYSICAL REVIEW, XXXVIII.

(To face page 216.)

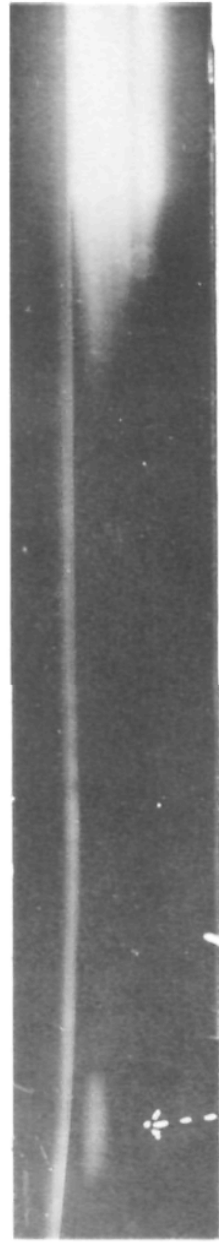

6

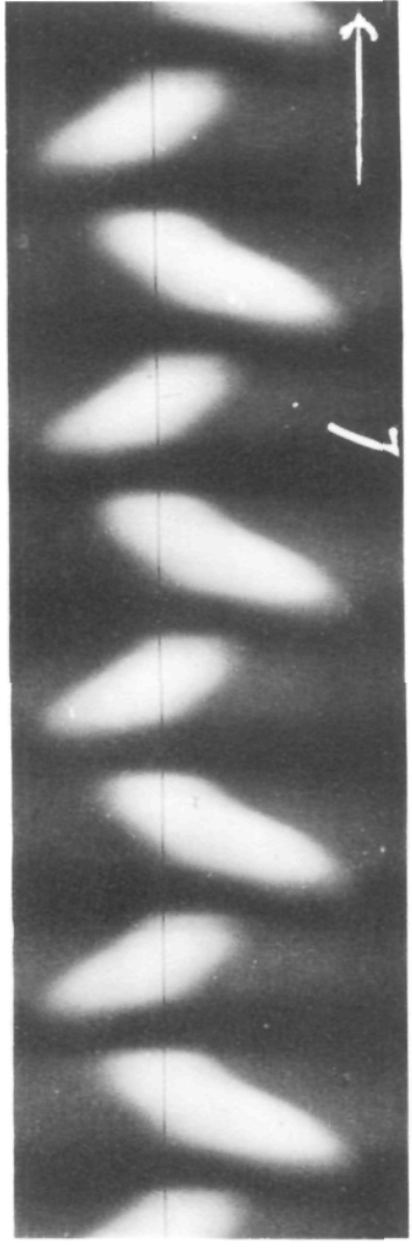

7
PLATE III.

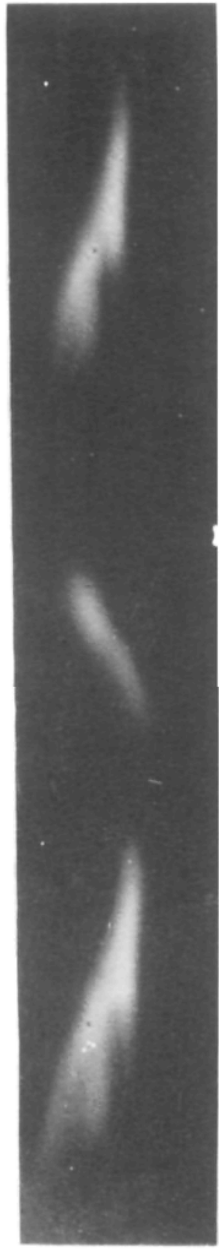

8

BROWN. A PHOTOGRAPHIC STUDY OF THE ELECTRIC ARC. 